\title{
Mixed Functionals of Convex Bodies
}

\section{R. Schneider}

Mathematisches Institut, Albert-Ludwigs-Universität, Eckerstrasse 1, D-79104 Freiburg i. Br., Germany

rschnei@ruf.uni-freiburg.de

\begin{abstract}
We define a class of real functions on tuples of convex bodies. They are a common generalization of mixed volumes and of certain functionals which have been studied in translative integral geometry. For polytopes, these functionals have various explicit representations in terms of volumes of lower-dimensional faces. For the mentioned functionals from integral geometry, these representations generalize a result of Weil and answer a question posed by Janson.
\end{abstract}

\section{Introduction}

It is a basic fact of the Brunn-Minkowski theory that the volume of a Minkowski combination of convex bodies admits a polynomial expansion. If $K_{1}, \ldots, K_{k}$ are convex bodies in $n$-dimensional Euclidean space $\mathbb{E}^{n}$ and if $\lambda_{1}, \ldots, \lambda_{k}$ are positive real numbers, then the volume $V_{n}$ satisfies

$$
\begin{aligned}
& V_{n}\left(\lambda_{1} K_{1}+\cdots+\lambda_{k} K_{k}\right) \\
& \quad=\sum_{\substack{m_{1}, \ldots, m_{k}=0 \\
m_{1}+\cdots+m_{k}=n}}^{n} \lambda_{1}^{m_{1}} \cdots \lambda_{k}^{m_{k}}\left(\begin{array}{c}
n \\
m_{1} \cdots m_{k}
\end{array}\right) V(\underbrace{K_{1}, \ldots, K_{1}}_{m_{1}}, \ldots, \underbrace{K_{k}, \ldots, K_{k}}_{m_{k}}) .
\end{aligned}
$$

This defines the mixed volumes appearing as coefficients (up to multinomial factors). A similar polynomial expansion holds in translative integral geometry; it has the form

$$
\begin{gathered}
\int_{\mathbb{E}^{n}} \cdots \int_{\mathbb{E}^{n}} \chi\left(\left(\lambda_{1} K_{1}+x_{1}\right) \cap \cdots \cap\left(\lambda_{k-1} K_{k-1}+x_{k-1}\right) \cap \lambda_{k} K_{k}\right) d x_{1} \cdots d x_{k-1} \\
=\sum_{\substack{m_{1}, \ldots, m_{k}=0 \\
m_{1}+\cdots+m_{k}=(k-1) n}}^{n} \lambda_{1}^{m_{1}} \cdots \lambda_{k}^{m_{k}} V_{m_{1}, \ldots, m_{k}}^{(0)}\left(K_{1}, \ldots, K_{k}\right) .
\end{gathered}
$$


Here $\chi(K)=1$ for a nonempty convex body $K$ and $\chi(\emptyset)=0$. More general expansions of this type, with the Euler characteristic $\chi$ replaced by other functions, have been studied by Weil [11], [12]. In the following we view both types of expansions as special cases of a general result. The chosen approach has the advantage that in the case of polytopes it yields special representations for the mixed functionals occurring as coefficients, in terms of volumes of lower-dimensional faces. For the mixed volumes, such representations were obtained by Betke [2] for $k=2$ and by Schneider [10] in general. Here we derive similar representations for mixed functionals generalizing those appearing in (1) and (2). For the coefficients in (2), the existence of such representations was conjectured by Janson [7].

\section{General Mixed Functionals}

We start with two real vector spaces $X$ and $Y$, of dimensions $p$ and $q$, respectively, where $p>q \geq 1$. The space $X$ will be equipped with a scalar product $\langle\cdot, \cdot\rangle$, for convenience. We suppose that a surjective linear map $f: X \rightarrow Y$ is given. Let ker $f$ be the kernel of $f$ and let $L \subset X$ be its orthogonal complement. By $\pi: X \rightarrow L$ we denote the orthogonal projection onto $L$ and by $\tilde{f}:=f \mid L$ the restriction of $f$ to $L$. Then $\tilde{f}: L \rightarrow Y$ is an isomorphism, and we have the factorization $f=\tilde{f} \circ \pi$.

If $v \in L^{\perp} \backslash\{0\}$, we write

$$
L_{v}:=L+\mathbb{R} v, \quad L_{v}^{+}:=L+\mathbb{R}^{+} v
$$

where $\mathbb{R}^{+}$denotes the set of positive real numbers. Let $\pi_{v}: X \rightarrow L_{v}$ denote the orthogonal projection onto $L_{v}$.

Now let $P \subset X$ be a (convex) polytope. For a vector $u \in X \backslash\{0\}$, the set $F(P, u)$ is the support set of $P$ with outer normal vector $u$. (For notions from convex geometry that are not explained here, we refer to [9].) For $i \in\{0, \ldots, \operatorname{dim} P\}, \mathcal{F}_{i}(P)$ denotes the set of $i$-dimensional faces of $P$, and for a face $F$ of $P$, the set $N(P, F)$ is the normal cone of $P$ at its face $F$. Thus, $u \in N(P, F)$ if and only if either $u=0$ or $u$ is an outer normal vector of a supporting hyperplane of $P$ containing $F$.

We choose a vector $v \in X$ satisfying the conditions

$$
v \in L^{\perp} \backslash\{0\}, \quad \operatorname{dim} F(P, u) \leq q \quad \text { for all } \quad u \in L_{v}^{+} .
$$

We refer to these conditions as the selection rule. To see which vectors are excluded by this rule, let $v \in L^{\perp} \backslash\{0\}$ be a vector not satisfying (3). Thus there is a vector $u \in L_{v}^{+}$ such that $\operatorname{dim} F(P, u)>q$. With $F(P, u)=: F$ and $u=x+\lambda v$ with $x \in L$ and $\lambda>0$, we have $x+\lambda v \in N(P, F)$ and, therefore,

$$
v \in L^{\perp} \cap \operatorname{lin}(N(P, F) \cup L) .
$$

From $\operatorname{dim} F(P, u)>q$ it follows that $\operatorname{dim} N(P, F)<p-q$. Thus the right-hand side of (4) is a proper linear subspace of $L^{\perp}$. Hence, the selection rule is satisfied for all vectors of $L^{\perp}=\operatorname{ker} f$, except those in finitely many proper linear subspaces determined by $P$. 
Now let $v$ satisfy (3). We recall an argument used, in a special case, on p. 292 of [10]. Let $F^{\prime}$ be a $q$-face of the polytope $\pi_{v} P$ with an exterior normal vector $u \in L_{v}^{+}$. Then $F^{\prime}=\pi_{v} F$ for the face $F:=F(P, u)$ of $P$. It follows from (3) that $F$ is a $q$-face of $P$; it satisfies

$$
N(P, F) \cap L_{v}^{+} \neq \emptyset .
$$

Vice versa, each $q$-face $F$ of $P$ satisfying this condition maps under $\pi_{v}$ into a $q$-face $F^{\prime}$ of $\pi_{v} P$ with exterior normal vector in $L_{v}^{+}$. The projection $\pi$ maps $F^{\prime}$ into a $q$-polytope in $L$. The $q$-faces $F^{\prime}$ of $\pi_{v} P$ with an outer normal vector $u \in L_{v}^{+}$are the facets, relative to $L_{v}$, of the polytope $\pi_{v} P$ that have an outer normal vector $u$ with $\langle u, v\rangle>0$. It follows that all the projections $\pi F^{\prime}=\pi F$, where $F^{\prime}$ and $F$ are chosen as above, cover $\pi P$ and have pairwise disjoint relative interiors. If $V_{q}$ denotes the $q$-dimensional volume in $L$ (induced by the scalar product), we deduce that

$$
V_{q}(\pi P)=\sum_{*(v)} V_{q}(\pi F)
$$

Here $*(v)$ refers to the following summation rule: $\sum_{*(v)}$ extends over all $q$-faces $F$ of $P$ satisfying

$$
N(P, F) \cap L_{v}^{+} \neq \emptyset .
$$

If we apply the isomorphism $\tilde{f}: L \rightarrow Y$, we obtain from (5) the representation

$$
V_{q}(f(P))=\sum_{*(v)} V_{q}(f(F)),
$$

where $V_{q}$ denotes an affine volume in $Y$.

The foregoing will now be applied to the situation described by the following data:

- the $n$-dimensional Euclidean space $\mathbb{E}^{n}(n \geq 1)$,

- two integers $k, r$ with $k>r \geq 1$,

- a surjective linear map $f:\left(\mathbb{E}^{n}\right)^{k} \rightarrow\left(\mathbb{E}^{n}\right)^{r}$.

Let $P_{1}, \ldots, P_{k} \subset \mathbb{E}^{n}$ be polytopes and define $P:=P_{1} \times \cdots \times P_{k} \subset\left(\mathbb{E}^{n}\right)^{k}$. We let $X=\left(\mathbb{E}^{n}\right)^{k}, Y=\left(\mathbb{E}^{n}\right)^{r}$, and equip the space $X$ with the scalar product induced by a scalar product on $\mathbb{E}^{n}$. The space $Y$ is endowed with the affine volume $V_{r n}$ induced by the same scalar product. From (7), we get the formula

$$
V_{r n}\left(f\left(P_{1} \times \cdots \times P_{k}\right)\right)=\sum_{*(v)} V_{r n}(f(F)),
$$

where $v$ has now to be chosen according to the selection rule

$$
v \in \operatorname{ker} f, \quad v \neq 0, \quad \operatorname{dim} F\left(P_{1} \times \cdots \times P_{k}, u\right) \leq r n \quad \text { for } \quad u \in L_{v}^{+} .
$$

If $F$ is a face of $P$ for which $V_{r n}(f(F))$ appears in the right-hand side of (8) and is not zero, then $F=F_{1} \times \cdots \times F_{k}$ with faces $F_{i} \in \mathcal{F}_{m_{i}}\left(P_{i}\right)\left(m_{i} \in\{0, \ldots, n\}, i=1, \ldots, k\right)$ and $\operatorname{dim} F=r n$, hence

$$
m_{1}+\cdots+m_{k}=r n
$$


The summation rule (6) is equivalent to

$$
N\left(P_{1} \times \cdots \times P_{k}, F_{1} \times \cdots \times F_{k}\right) \cap L_{v}^{+} \neq \emptyset .
$$

The assumption $V_{r n}(f(F)) \neq 0$ implies

$$
\operatorname{dim} f\left(F_{1} \times \cdots \times F_{k}\right)=r n .
$$

Hence, the linear map $f$ restricted to the affine hull of $F_{1} \times \cdots \times F_{k}$ is injective. It follows that

$$
V_{r n}\left(f\left(F_{1} \times \cdots \times F_{k}\right)\right)=\varphi\left(f, \text { aff } F_{1}, \ldots, \text { aff } F_{k}\right) V_{m_{1}}\left(F_{1}\right) \cdots V_{m_{k}}\left(F_{k}\right)
$$

where $\varphi\left(f\right.$, aff $F_{1}, \ldots$, aff $\left.F_{k}\right)$ is the factor by which $f \mid \operatorname{aff}\left(F_{1} \times \cdots \times F_{k}\right)$ distorts the $r n$-dimensional volume. Here $V_{m}$ denotes the $m$-dimensional volume in $m$-dimensional affine subspaces of $\mathbb{E}^{n}$. For $\lambda_{1}, \ldots, \lambda_{k}>0$ we deduce that

$$
V_{r n}\left(f\left(\lambda_{1} F_{1} \times \cdots \times \lambda_{k} F_{k}\right)\right)=\lambda_{1}^{m_{1}} \cdots \lambda_{k}^{m_{k}} \varphi\left(f, \text { aff } F_{1}, \ldots, \text { aff } F_{k}\right) V_{m_{1}}\left(F_{1}\right) \cdots V_{m_{k}}\left(F_{k}\right)
$$

Together with (8), this gives

$$
V_{r n}\left(f\left(\lambda_{1} P_{1} \times \cdots \times \lambda_{k} P_{k}\right)\right)=\sum_{\substack{m_{1}, \ldots, m_{k}=0 \\ m_{1}+\cdots+m_{k}=r n}}^{n} \lambda_{1}^{m_{1}} \cdots \lambda_{k}^{m_{k}} V_{m_{1}, \ldots, m_{k}}^{(f)}\left(P_{1}, \ldots, P_{k}\right)
$$

with

$$
V_{m_{1}, \ldots, m_{k}}^{(f)}\left(P_{1}, \ldots, P_{k}\right):=\sum_{*(v)} \varphi\left(f, \text { aff } F_{1}, \ldots, \text { aff } F_{k}\right) V_{m_{1}}\left(F_{1}\right) \cdots V_{m_{k}}\left(F_{k}\right)
$$

Here the summation rule $*(v)$ now indicates that, for given $m_{1}, \ldots, m_{k} \in\{0, \ldots, n\}$ satisfying (10), the sum extends over all $k$-tuples $\left(F_{1}, \ldots, F_{k}\right)$ of faces $F_{i} \in \mathcal{F}_{m_{i}}\left(P_{i}\right)$ $(i=1, \ldots, k)$ satisfying $(11)$.

By approximation and continuity, it follows from (13) that for arbitrary convex bodies $K_{1}, \ldots, K_{k} \subset \mathbb{E}^{n}$ one has a polynomial expansion

$$
V_{r n}\left(f\left(\lambda_{1} K_{1} \times \cdots \times \lambda_{k} K_{k}\right)\right)=\sum_{\substack{m_{1}, \ldots, m_{k}=0 \\ m_{1}+\cdots+m_{k}=r n}}^{n} \lambda_{1}^{m_{1}} \cdots \lambda_{k}^{m_{k}} V_{m_{1}, \ldots, m_{k}}^{(f)}\left(K_{1}, \ldots, K_{k}\right)
$$

for $\lambda_{1}, \ldots, \lambda_{k}>0$, with uniquely determined coefficients $V_{m_{1}, \ldots, m_{k}}^{(f)}\left(K_{1}, \ldots, K_{k}\right)$. We call each function $V_{m_{1}, \ldots, m_{k}}^{(f)}$ a mixed functional induced by $f$.

As a function of its $i$ th argument, $V_{m_{1}, \ldots, m_{k}}^{(f)}$ has the following properties: it is translation invariant, positively homogeneous of degree $m_{i}$, and a valuation, that is, it satisfies

$$
\begin{aligned}
V_{m_{1}, \ldots, m_{k}}^{(f)}( & \left.K_{1}, \ldots, K_{i}^{\prime} \cup K_{i}^{\prime \prime}, \ldots, K_{k}\right)+V_{m_{1}, \ldots, m_{k}}^{(f)}\left(K_{1}, \ldots, K_{i}^{\prime} \cap K_{i}^{\prime \prime}, \ldots, K_{k}\right) \\
& =V_{m_{1}, \ldots, m_{k}}^{(f)}\left(K_{1}, \ldots, K_{i}^{\prime}, \ldots, K_{k}\right)+V_{m_{1}, \ldots, m_{k}}^{(f)}\left(K_{1}, \ldots, K_{i}^{\prime \prime}, \ldots, K_{k}\right)
\end{aligned}
$$


for convex bodies $K_{i}^{\prime}, K_{i}^{\prime \prime}$ such that $K_{i}^{\prime} \cup K_{i}^{\prime \prime}$ is convex. The first property is clear from the definition, the second follows from (14) and approximation, and the third is obtained as follows. Let $K_{i}^{\prime}, K_{i}^{\prime \prime}$ be convex bodies such that $K_{i}^{\prime} \cup K_{i}^{\prime \prime}$ is convex. In (15) we replace $K_{i}$ by $K_{i}^{\prime} \cup K_{i}^{\prime \prime}$ and by $K_{i}^{\prime} \cap K_{i}^{\prime \prime}$ and add the two expressions. The result is then obtained by comparing coefficients, if we use the valuation property of the volume and the following lemma.

Lemma. Let $X, Y$ be real vector spaces and let $f: X \rightarrow Y$ be a linear map. If $K, L \subset X$ are convex sets such that $K \cup L$ is convex, then $f(K \cap L)=f(K) \cap f(L)$.

Proof. Let $y \in f(K) \cap f(L)$. Then $y=f\left(x_{1}\right)=f\left(x_{2}\right)$ with suitable $x_{1} \in K, x_{2} \in L$. Since $K \cup L$ is convex, there is a number $\lambda \in[0,1]$ such that $(1-\lambda) x_{1}+\lambda x_{2} \in$ $K \cap L$. The linearity of $f$ gives $f\left((1-\lambda) x_{1}+\lambda x_{2}\right)=(1-\lambda) f\left(x_{1}\right)+\lambda f\left(x_{2}\right)=y$, thus $y \in f(K \cap L)$. Since $f(K \cap L) \subset f(K) \cap f(L)$ holds trivially, the assertion follows.

\section{Special Cases}

First we recall the special case treated in [10], which leads to mixed volumes. Here $k>1, r=1$, and the linear map $f:\left(\mathbb{E}^{n}\right)^{k} \rightarrow \mathbb{E}^{n}$ is defined by

$$
f\left(x_{1}, \ldots, x_{k}\right):=x_{1}+\cdots+x_{k} .
$$

A comparison of (15) with formula (5.1.26) in [9] shows that

$$
V_{m_{1}, \ldots, m_{k}}^{(f)}\left(K_{1}, \ldots, K_{k}\right)=\left(\begin{array}{c}
n \\
m_{1} \cdots m_{k}
\end{array}\right) V\left(K_{1}\left[m_{1}\right], \ldots, K_{k}\left[m_{k}\right]\right)
$$

where

$$
V\left(K_{1}\left[m_{1}\right], \ldots, K_{k}\left[m_{k}\right]\right):=V(\underbrace{K_{1}, \ldots, K_{1}}_{m_{1}}, \ldots, \underbrace{K_{k}, \ldots, K_{k}}_{m_{k}})
$$

and $V$ on the right side denotes the mixed volume. The point is that this approach leads to special explicit formulas for mixed volumes of polytopes in terms of volumes of faces. If the selection and summation rules and the factor $\varphi\left(f\right.$, aff $F_{1}, \ldots$, aff $\left.F_{k}\right)$ are interpreted for the chosen function $f$, one obtains the following result, as formulated on p. 295 of [10] and p. 263 of [5]. In the following, [ $F_{1}, \ldots, F_{k}$ ] denotes the volume of the parallelepiped that is the sum of unit cubes in the affine hulls of $F_{1}, \ldots, F_{k}$. (Observe that $\left[F_{1}, \ldots, F_{k}\right]=0$ if $\operatorname{dim}\left(F_{1}+\cdots+F_{k}\right)<n$.)

Theorem 1. Let $P_{1}, \ldots, P_{k} \subset \mathbb{E}^{n}$ be polytopes $(k \geq 2)$. Let $v_{1}, \ldots, v_{k} \in \mathbb{E}^{n}$ be such that

$$
v_{1}+\cdots+v_{k}=0 \quad\left(v_{1}, \ldots, v_{k}\right) \neq(0, \ldots, 0)
$$

and

$$
\bigcap_{i=1}^{k}\left(\operatorname{relint} N\left(P_{i}, F_{i}\right)-v_{i}\right)=\emptyset
$$


whenever $F_{i}$ is a face of $P_{i}$ and $\operatorname{dim} F_{1}+\cdots+\operatorname{dim} F_{k}>n$. Then

$$
\left(\begin{array}{c}
n \\
m_{1} \cdots m_{k}
\end{array}\right) V\left(P_{1}\left[m_{1}\right], \ldots, P_{k}\left[m_{k}\right]\right)=\sum\left[F_{1}, \ldots, F_{k}\right] V_{m_{1}}\left(F_{1}\right) \cdots V_{m_{k}}\left(F_{k}\right),
$$

where the summation extends over the $k$-tuples $\left(F_{1}, \ldots, F_{k}\right)$ of $m_{i}$-faces $F_{i}$ of $P_{i}$ with

$$
\bigcap_{i=1}^{k}\left(N\left(P_{i}, F_{i}\right)-v_{i}\right) \neq \emptyset
$$

An application of this result in computational convexity appears in [4]. We have quoted the result here to facilitate comparison with the new result to which we turn now.

For convex bodies $K_{1}, \ldots, K_{k} \subset \mathbb{E}^{n}(k \geq 2)$, we consider the integral

$$
I\left(K_{1}, \ldots, K_{k}\right):=\int_{\mathbb{E}^{n}} \cdots \int_{\mathbb{E}^{n}} \chi\left(\left(K_{1}+x_{1}\right) \cap \cdots \cap\left(K_{k-1}+x_{k-1}\right) \cap K_{k}\right) d x_{1} \cdots d x_{k-1},
$$

where $\chi$ denotes the Euler characteristic and $d x_{i}$ indicates integration with respect to Lebesgue measure on $\mathbb{E}^{n}$. The study of such integrals is a task of the integral geometry for the translation group. More general integrals of this type, with the Euler characteristic replaced by curvature measures, have been investigated by Weil [11], [12], with a view to applications in the stochastic geometry of stationary structures.

If we define the set

$$
D\left(K_{1}, \ldots, K_{k}\right):=\left\{\left(x_{1}, \ldots, x_{k-1}\right) \in\left(\mathbb{E}^{n}\right)^{k-1}:\left(K_{1}+x_{1}\right) \cap \cdots \cap\left(K_{k-1}+x_{k-1}\right) \cap K_{k} \neq \emptyset\right\},
$$

then

$$
I\left(K_{1}, \ldots, K_{k}\right)=V_{(k-1) n}\left(D\left(K_{1}, \ldots, K_{k}\right)\right)
$$

The functional

$$
K \mapsto V_{(k-1) n}(D(\underbrace{K, \ldots, K}_{k}))
$$

was investigated in [8].

Now let $f:\left(\mathbb{E}^{n}\right)^{k} \rightarrow\left(\mathbb{E}^{n}\right)^{k-1}$ be the linear map given by

$$
f\left(x_{1}, \ldots, x_{k}\right):=\left(x_{k}-x_{1}, \ldots, x_{k}-x_{k-1}\right) .
$$

Then $f\left(K_{1} \times \cdots \times K_{k}\right)=D\left(K_{1}, \ldots, K_{k}\right)$. Therefore, the above theory applies, and from (15) we obtain the polynomial expansion

$$
\begin{gathered}
\int_{\mathbb{E}^{n}} \cdots \int_{\mathbb{E}^{n}} \chi\left(\left(\lambda_{1} K_{1}+x_{1}\right) \cap \cdots \cap\left(\lambda_{k-1} K_{k-1}+x_{k-1}\right) \cap \lambda_{k} K_{k}\right) d x_{1} \cdots d x_{k-1} \\
=\sum_{\substack{m_{1}, \ldots, m_{k}=0 \\
m_{1}+\cdots+m_{k}=(k-1) n}}^{n} \lambda_{1}^{m_{1}} \cdots \lambda_{k}^{m_{k}} V_{m_{1}, \ldots, m_{k}}^{(0)}\left(K_{1}, \ldots, K_{k}\right) .
\end{gathered}
$$

Here we have written $V_{m_{1}, \ldots, m_{k}}^{(0)}:=V_{m_{1}, \ldots, m_{k}}^{(f)}$; this notation is consistent with the one used by Weil [11], [12]. 
Similarly as the mixed volumes given by (16), the coefficients appearing here have a symmetry property: if $\sigma$ is a permutation of $\{1, \ldots, k\}$, then

$$
V_{m_{\sigma(1)}, \ldots, m_{\sigma(k)}}^{(0)}\left(K_{\sigma(1)}, \ldots, K_{\sigma(k)}\right)=V_{m_{1}, \ldots, m_{k}}^{(0)}\left(K_{1}, \ldots, K_{k}\right) .
$$

This follows from the fact that $D\left(K_{\sigma(1)}, \ldots, K_{\sigma(k)}\right)$ is obtained from $D\left(K_{1}, \ldots, K_{k}\right)$ by a volume-preserving linear transformation.

In order to obtain explicit representations of the functionals $V_{m_{1}, \ldots, m_{k}}^{(0)}$ for polytopes, we now interpret, for the function $f$ given by (17), the selection and summation rules.

First we note that

$$
\left(v_{1}, \ldots, v_{k}\right) \in L^{\perp}=\operatorname{ker} f \Leftrightarrow v_{1}=\cdots=v_{k}
$$

and

$$
\left(x_{1}, \ldots, x_{k}\right) \in L \quad \Leftrightarrow \quad x_{1}+\cdots+x_{k}=0 .
$$

Thus the vector $v \in\left(\mathbb{E}^{n}\right)^{k}$, which we have to choose in agreement with the selection rule (3), is of the form $v=(w, \ldots, w)$ with $w \in \mathbb{E}^{n} \backslash\{0\}$. According to the selection rule, the vector $w$ has to satisfy

$$
\operatorname{dim} F\left(P_{1} \times \cdots \times P_{k}, u\right) \leq(k-1) n \quad \text { for all } \quad u \in L_{v}^{+} .
$$

The vector $u=\left(u_{1}, \ldots, u_{k}\right)$ is in $L_{v}^{+}$if and only if

$u_{i}=x_{i}+\lambda w \quad$ with $\quad x_{i} \in \mathbb{E}^{n} \quad(i=1, \ldots, k), \quad x_{1}+\cdots+x_{k}=0, \quad$ and $\quad \lambda \in \mathbb{R}^{+}$,

hence if and only if $u_{1}+\cdots+u_{k}=\mu w$ with $\mu>0$. Since

$$
F\left(P_{1} \times \cdots \times P_{k},\left(u_{1}, \ldots, u_{k}\right)\right)=F\left(P_{1}, u_{1}\right) \times \cdots \times F\left(P_{k}, u_{k}\right)
$$

(with $\left.F\left(P_{i}, 0\right):=P_{i}\right)$, condition (19) is equivalent to

$$
\operatorname{dim} F\left(P_{1}, u_{1}\right)+\cdots+\operatorname{dim} F\left(P_{k}, u_{k}\right) \leq(k-1) n
$$

whenever $u_{i} \in \mathbb{E}^{n}$ and $u_{1}+\cdots+u_{k}=\mu w$ with $\mu>0$. This condition can be reformulated as follows. If $F_{i}$ is a face of $P_{i}$ for $i=1, \ldots, k$ and if

$$
\operatorname{dim} F_{1}+\cdots+\operatorname{dim} F_{k}>(k-1) n,
$$

then

$$
w \notin \sum_{i=1}^{k} \operatorname{relint} N\left(P_{i}, F_{i}\right)
$$

(since $F_{i}=F\left(P, u_{i}\right)$ holds if and only if $u_{i} \in \operatorname{relint} N\left(P_{i}, F_{i}\right)$ ). Suppose that $w \in$ $\mathbb{E}^{n} \backslash\{0\}$ has been chosen so that this is satisfied. By the summation rule, the sum $\sum_{*(v)}$ in (14) extends over all $k$-tuples $\left(F_{1}, \ldots, F_{k}\right)$ with $F_{i} \in \mathcal{F}_{m_{i}}(P)(i=1, \ldots, k)$ and with

$$
N\left(P_{1} \times \cdots \times P_{k}, F_{1} \times \cdots \times F_{k}\right) \cap L_{v}^{+} \neq \emptyset .
$$


The latter is equivalent to the existence of a vector

$$
\left(u_{1}, \ldots, u_{k}\right) \in N\left(P_{1} \times \cdots \times P_{k}, F_{1} \times \cdots \times F_{k}\right)
$$

with $u_{1}+\cdots+u_{k}=\mu w$ for some $\mu>0$. Since (22) holds if and only if $u_{i} \in N\left(P_{i}, F_{i}\right)$ for $i=1, \ldots, k$, we see that $(21)$ is equivalent to

$$
w \in \sum_{i=1}^{k} N\left(P_{i}, F_{i}\right)
$$

It remains to determine the factor $\varphi$ in (14). For this, we need an extension of the function $[\cdot, \ldots, \cdot]$. Let $L_{1}, \ldots, L_{k}$ be linear subspaces of $\mathbb{E}^{n}$ with $\sum_{i=1}^{k} \operatorname{dim} L_{i}=m \leq n$. We choose an orthonormal basis in each $L_{i}$ (the empty set if $\operatorname{dim} L_{i}=0$ ) and let $\operatorname{det}\left(L_{1}, \ldots, L_{k}\right)$ be the $m$-dimensional volume of the parallelepiped spanned by these vectors. Next we define

$$
\left[L_{1}, \ldots, L_{k}\right]:=\operatorname{det}\left(L_{1}^{\perp}, \ldots, L_{k}^{\perp}\right) \quad \text { if } \quad \sum_{i=1}^{k} \operatorname{dim} L_{i} \geq(k-1) n
$$

and $\left[L_{1}, \ldots, L_{k}\right]:=0$ if $\sum_{i=1}^{k} \operatorname{dim} L_{i}<(k-1) n$ (see [12] for some properties of this function). For subsets $F_{1}, \ldots, F_{k} \subset \mathbb{E}^{n}$ we then define $\left[F_{1}, \ldots, F_{k}\right]:=\left[L_{1}, \ldots, L_{k}\right]$, where $L_{i}$ is the linear subspace parallel to the affine hull of $F_{i}(i=1, \ldots, k)$.

Now let $m_{1}, \ldots, m_{k} \in\{0, \ldots, n\}$ with $m_{1}+\cdots+m_{k}=(k-1) n$ be given and choose a polytope $F_{i} \subset \mathbb{E}^{n}$ of dimension $m_{i}(i=1, \ldots, k)$. Then from (18) and (14), with a suitably chosen vector $v$, we get

$$
\begin{gathered}
\int_{\mathbb{E}^{n}} \cdots \int_{\mathbb{E}^{n}} \chi\left(\left(F_{1}+x_{1}\right) \cap \cdots \cap\left(F_{k-1}+x_{k-1}\right) \cap F_{k}\right) d x_{1} \cdots d x_{k-1} \\
=\varphi\left(f, \text { aff } F_{1}, \ldots, \text { aff } F_{k}\right) V_{m_{1}}\left(F_{1}\right) \cdots V_{m_{k}}\left(F_{k}\right) .
\end{gathered}
$$

By elementary integration (or see Proposition 2 of [12] for $j=0$ ), the left side is equal to $\left[F_{1}, \ldots, F_{k}\right] V_{m_{1}}\left(F_{1}\right) \cdots V_{m_{k}}\left(F_{k}\right)$. Thus we arrive at the following result.

Theorem 2. Let $P_{1}, \ldots, P_{k} \subset \mathbb{E}^{n}$ be polytopes $(k \geq 2)$. Let $w \in \mathbb{E}^{n} \backslash\{0\}$ be such that

$$
w \notin \sum_{i=1}^{k} \operatorname{relint} N\left(P_{i}, F_{i}\right)
$$

whenever $F_{i}$ is a face of $P_{i}$ and $\operatorname{dim} F_{1}+\cdots+\operatorname{dim} F_{k}>(k-1) n$. Then

$$
V_{m_{1}, \ldots, m_{k}}^{(0)}\left(P_{1}, \ldots, P_{k}\right)=\sum\left[F_{1}, \ldots, F_{k}\right] V_{m_{1}}\left(F_{1}\right) \cdots V_{m_{k}}\left(F_{k}\right),
$$

where the summation extends over the k-tuples $\left(F_{1}, \ldots, F_{k}\right)$ of $m_{i}$-faces $F_{i}$ of $P_{i}$ with

$$
w \in \sum_{i=1}^{k} N\left(P_{i}, F_{i}\right)
$$


The representation (25) is more general than the one given by Weil [11], [12]. To see this, we write (25) in the form

$$
\begin{aligned}
V_{m_{1}, \ldots, m_{k}}^{(0)} & \left(P_{1}, \ldots, P_{k}\right) \\
& =\sum_{F_{1} \in \mathcal{F}_{m_{1}}\left(P_{1}\right)} \cdots \sum_{F_{k} \in \mathcal{F}_{m_{k}}\left(P_{k}\right)} \mathbf{1}_{A}(w)\left[F_{1}, \ldots, F_{k}\right] V_{m_{1}}\left(F_{1}\right) \cdots V_{m_{k}}\left(F_{k}\right),
\end{aligned}
$$

where $A:=\sum_{i=1}^{k} N\left(P_{i}, F_{i}\right)$ and $\mathbf{1}_{A}$ denotes the indicator function of $A$. Let $\lambda_{\mathrm{s}}$ denote the normalized spherical Lebesgue measure on the unit sphere $S^{n-1}$ of $\mathbb{E}^{n}$. Equality (27) holds for $\lambda_{\mathrm{s}}$-almost all $w \in S^{n-1}$, since condition (24) excludes only a set of unit vectors $w$ of $\lambda_{\mathrm{s}}$-measure zero. Hence, integration of (27) over $S^{n-1}$ gives

$$
\begin{aligned}
& V_{m_{1}, \ldots, m_{k}}^{(0)}\left(P_{1}, \ldots, P_{k}\right)=\sum_{F_{1} \in \mathcal{F}_{m_{1}}\left(P_{1}\right)} \ldots \sum_{F_{k} \in \mathcal{F}_{m_{k}}\left(P_{k}\right)} \lambda_{\mathrm{s}}\left(S^{n-1} \cap \sum_{i=1}^{k} N\left(P_{i}, F_{i}\right)\right) \\
& \text { - }\left[F_{1}, \ldots, F_{k}\right] V_{m_{1}}\left(F_{1}\right) \cdots V_{m_{k}}\left(F_{k}\right) \text {. }
\end{aligned}
$$

The common exterior angle $\gamma\left(F_{1}, \ldots, F_{k} ; P_{1}, \ldots, P_{k}\right)$ is defined by

$$
\gamma\left(F_{1}, \ldots, F_{k} ; P_{1}, \ldots, P_{k}\right):=\gamma\left(\bigcap_{i=1}^{k}\left(F_{i}-x_{i}\right), \bigcap_{i=1}^{k}\left(P_{i}-x_{i}\right)\right),
$$

where $x_{i} \in \operatorname{relint} F_{i}$ for $i=1, \ldots, k$ can be chosen arbitrarily and where $\gamma(F, P)$ on the right denotes the exterior angle of $P$ at $F$ (see p. 100 of [9]). Now

$$
N\left(\bigcap_{i=1}^{k}\left(P_{i}-x_{i}\right), \bigcap_{i=1}^{k}\left(F_{i}-x_{i}\right)\right)=\sum_{i=1}^{k} N\left(P_{i}-x_{i}, F_{i}-x_{i}\right)=\sum_{i=1}^{k} N\left(P_{i}, F_{i}\right)
$$

by Theorem 2.2.1(b) of [9], hence

$$
\begin{aligned}
V_{m_{1}, \ldots, m_{k}}^{(0)}\left(P_{1}, \ldots, P_{k}\right)= & \sum_{F_{1} \in \mathcal{F}_{m_{1}}\left(P_{1}\right)} \ldots \sum_{F_{k} \in \mathcal{F}_{m_{k}}\left(P_{k}\right)} \gamma\left(F_{1}, \ldots, F_{k} ; P_{1}, \ldots, P_{k}\right) \\
& \cdot\left[F_{1}, \ldots, F_{k}\right] V_{m_{1}}\left(F_{1}\right) \cdots V_{m_{k}}\left(F_{k}\right) .
\end{aligned}
$$

This is the representation obtained by Weil [11], [12]

Among the mixed functionals $V_{m_{1}, \ldots, m_{k}}^{(0)}$, the one with $k=n$ and $m_{1}=\cdots=m_{n}=$ $n-1$ is of particular interest. We write

$$
\beta\left(K_{1}, \ldots, K_{n}\right):=V_{n-1, \ldots, n-1}^{(0)}\left(K_{1}, \ldots, K_{n}\right)
$$

for convex bodies $K_{1}, \ldots, K_{n}$; then

$$
\begin{aligned}
\beta\left(K_{1}, \ldots, K_{n}\right) & \\
& =\int_{S^{n-1}} \cdots \int_{S^{n-1}} \mathbf{1}_{\operatorname{pos}\left\{u_{1}, \ldots, u_{n}\right\}}(w)\left[u_{1}, \ldots, u_{n}\right] d S_{n-1}\left(K_{1}, u_{1}\right) \cdots d S_{n-1}\left(K_{n}, u_{n}\right) \\
& =\int_{S^{n-1}} \cdots \int_{S^{n-1}} \gamma\left(u_{1}, \ldots, u_{n}\right)\left[u_{1}, \ldots, u_{n}\right] d S_{n-1}\left(K_{1}, u_{1}\right) \cdots d S_{n-1}\left(K_{n}, u_{n}\right),
\end{aligned}
$$


where $\gamma\left(u_{1}, \ldots, u_{n}\right):=\lambda_{\mathrm{s}}\left(S^{n-1} \cap \operatorname{pos}\left\{u_{1}, \ldots, u_{n}\right\}\right)$, where $\left[u_{1}, \ldots, u_{n}\right]$ denotes the volume of the parallelepiped spanned by the vectors $u_{1}, \ldots, u_{n}$, and $S_{n-1}(K, \cdot)$ is the area measure of the convex body $K$ (see p. 203 of [8]). If $K_{1}, \ldots, K_{n}$ are polytopes, (29) and (30) are rewritten versions of (27) and (28), and the general result follows by approximation, using the weak continuity of the area measures. The functional $\beta$ was first introduced (for $n=3$ and with different notation) by Blaschke [3, see (53)] and Berwald and Varga [1, see (20.4)]. The function $K \mapsto \beta(K, \ldots, K)$ plays an important role in Janson's [6] investigation on random coverings by homothets of a convex body. Janson introduced a functional $\beta\left(K_{1}, \ldots, K_{n}, w\right)$ by an integral equivalent to (29) and later showed (Corollary 7.4) that it is, for $\lambda_{s}$-almost all $w \in S^{n-1}$, independent of the vector $w$. He obtained this fact as a consequence of a deeper asymptotic result on random coverings, and posed the question (p. 97) for a direct proof of this fact of integral geometry. Such a direct proof is given above.

From (30), we can deduce another representation of $\beta\left(K_{1}, \ldots, K_{n}\right)$ in the case where $K_{1}, \ldots, K_{n}$ are centrally symmetric, namely,

$$
V_{n-1, \ldots, n-1}^{(0)}\left(K_{1}, \ldots, K_{n}\right)=\frac{n !}{2^{n}} V\left(\Pi K_{1}, \ldots, \Pi K_{n}\right) .
$$

Here $\Pi K$ denotes the projection body of $K$ (see p. 296 of [9]). This formula, which is due to Weil [11, Corollary 4.3], can also easily be obtained as follows. We have

$$
\sum_{\varepsilon_{i} \in\{1,-1\}} \gamma\left(\varepsilon_{1} u_{1}, \ldots, \varepsilon_{n} u_{n}\right)=1
$$

and if $K_{1}$ is centrally symmetric, then $d S_{n-1}\left(K_{i}, \varepsilon_{i} u_{i}\right)=d S_{n-1}\left(K_{i}, u_{i}\right)$. Hence, (30) gives

$$
\begin{aligned}
V_{n-1, \ldots, n-1}^{(0)}( & \left.K_{1}, \ldots, K_{n}\right) \\
= & \frac{1}{2^{n}} \int_{S^{n-1}} \cdots \int_{S^{n-1}}\left[u_{1}, \ldots, u_{n}\right] d S_{n-1}\left(K_{1}, u_{1}\right) \cdots d S_{n-1}\left(K_{n}, u_{n}\right),
\end{aligned}
$$

and (31) follows from (5.3.35) of [9].

This argument also works if merely $n-1$ of the bodies $K_{1}, \ldots, K_{n}$ are centrally symmetric, since

$$
\sum_{\varepsilon_{i} \in\{1,-1\}} \gamma\left(\varepsilon_{1} u_{1}, \ldots, \varepsilon_{n-1} u_{n-1}, u_{n}\right)=\frac{1}{2}
$$

As a final remark, we wish to point out that the mixed functionals $V_{m_{1}, \ldots, m_{k}}^{(0)}$ give rise to interesting extremal problems. Considering only the case of identical arguments, we note that the functional given by

$$
F_{m_{1}, \ldots, m_{k}}(K):=V_{m_{1}, \ldots, m_{k}}^{(0)}(K, \ldots, K) / V_{n}(K)^{k-1}
$$

is continuous and invariant under affine transformations, hence, on the space $\mathcal{K}_{0}^{n}$ of convex bodies with interior points it attains a maximum and a minimum. Only the cases $1 \leq m_{1}, \ldots, m_{k} \leq n-1$ are of interest here, since $V_{n, m_{2}, \ldots, m_{k}}^{(0)}\left(K_{1}, \ldots, K_{n}\right)=$ 
$V_{n}\left(K_{1}\right) V_{m_{2}, \ldots, m_{k}}^{(0)}\left(K_{2}, \ldots, K_{n}\right)$ (as follows from (25) and approximation, or from Corollary 3.3 of [11]), and if $m_{i} \leq n-1$ for $i=1, \ldots, k$, the condition $\sum_{i=1}^{k} m_{i}=(k-1) n$ implies $m_{i} \geq 1$ (and $k \leq n$ ). The case $k=2$ leads to mixed volumes, since

$$
V_{m, n-m}^{(0)}(K, M)=\left(\begin{array}{c}
n \\
m
\end{array}\right) V(K[m],-M[n-m]) .
$$

This settles the two-dimensional case: for $n=2$, only the functional $F_{1,1}$ is of interest, and

$$
1 \leq F_{1,1}(K)=\frac{V(K,-K)}{V_{2}(K)} \leq 2
$$

(see Theorem 7.3.1 of [9], where $V_{2}(\mathrm{D} K)=2 V(K,-K)+2 V_{2}(K)$ ). Equality on the left holds precisely if $K$ is centrally symmetric, and on the right precisely if $K$ is a triangle.

It was proved in [8] that $V_{p n}(D(K, \ldots, K)) / V_{n}(K)^{p}$ is maximal if and only if $K$ is a simplex, for each $p \in \mathbb{N}$. From this inequality (for $p \rightarrow \infty$ ), Janson [7] deduced that

$$
F_{n-1, \ldots, n-1}(K) \leq n^{n},
$$

with equality if $K$ is a simplex. It is not known whether this is the only case of equality. One may conjecture (as partially suggested in [6]-[8]) that each functional $F_{m_{1}, \ldots, m_{k}}$ attains its maximum on simplices and, for $k \geq 3$, its minimum on ellipsoids, and only on these bodies. This conjecture will probably be difficult to decide, since it includes two long-standing open problems. First, by (32), the maximum of $F_{m, n-m}$ is the subject of a question of Godbersen from 1938, which is still unanswered (see Note 3 on p. 412 of [9]). Second, if $K_{1}, \ldots, K_{n} \in \mathcal{K}_{0}^{n}$ are centrally symmetric, then (31) holds, hence

$$
F_{n-1, \ldots, n-1}(K)=\frac{n !}{2^{n}} \frac{V_{n}(\Pi K)}{V_{n}(K)^{n-1}} .
$$

Therefore, the question for the minimum of $F_{n-1, \ldots, n-1}$ on centrally symmetric bodies leads to Petty's unsolved problem on the volume of projection bodies (see p. 415 of [9]).

\section{References}

1. Berwald, L., and Varga, O., Integralgeometrie 24. Über die Schiebungen im Raum. Math. Z. 42 (1937), 710-736.

2. Betke, U., Mixed volumes of polytopes. Arch. Math. 58 (1992), 388-391.

3. Blaschke, W., Integralgeometrie 21. Über Schiebungen. Math. Z. 42 (1937), 399-410.

4. Dyer, M., Gritzmann, P., and Hufnagel, A., On the complexity of computing mixed volumes. SIAM J. Comput. 27 (1998), 356-400.

5. Henk, M., Richter-Gebert, J., and Ziegler, G. M., Basic properties of convex polytopes. In: Handbook of Discrete and Computational Geometry (J. E. Goodman and J. O'Rourke, eds.). CRC Press, Boca Raton, FL, 1997, pp. 243-270.

6. Janson, S., Random coverings in several dimensions. Acta Math. 156 (1986), 83-118.

7. Janson, S., Letter to the author. September 1996.

8. Schneider, R., Eine Verallgemeinerung des Differenzenkörpers. Monatsh. Math. 74 (1970), 258-272.

9. Schneider, R., Convex Bodies: the Brunn-Minkowski Theory. Encyclopedia of Mathematics and Its Applications, vol. 44. Cambridge University Press, Cambridge, 1993. 
10. Schneider, R., Polytopes and Brunn-Minkowski theory. In: Polytopes: Abstract, Convex and Computational (Scarborough, 1993; T. Bisztriczky, P. McMullen, R. Schneider, and A. Ivić Weiss, eds.). NATO ASI Series C, vol. 440. Kluwer, Dordrecht, 1994, pp. 273-299.

11. Weil, W., Iterations of translative integral formulae and non-isotropic Poisson processes of particles. Math. Z. 205 (1990), 531-549.

12. Weil, W., Mixed measures and functionals of translative integral geometry. Preprint 98/21, Fakultät für Mathematik, Universität Karlsruhe.

Received January 5, 1999, and in revised form March 12, 1999. Online publication May 16, 2000. 